\title{
The Extreme Tail of the Non-Gaussian Mass Function
}

\author{
Aseem Paranjape* \\ Abdus Salam ICTP, Strada Costiera 11, 34151 Trieste, Italy \\ Christopher Gordon $\dagger$ \\ Oxford Astrophysics, Physics, DWB, \\ Keble Road, Oxford, OX1 3RH, UK \\ Shaun Hotchkiss \\ Department of Physics, University of Helsinki and Helsinki Institute of Physics, \\ P.O. Box 64, FIN-00014 University of Helsinki, Finland
}

\begin{abstract}
Number counts of massive high-redshift clusters provide a window to study primordial nonGaussianity. The current quality of data, however, forces the statistical analysis to probe a region of parameter space - the extreme tail of the mass function - which is neither accessible in any of the currently available theoretical prescriptions for calculating the mass function, nor calibrated in $N$-body simulations. In this work we present a new analytical prescription for calculating a "resummed" non-Gaussian halo mass function, which is constructed to remain stable in the extreme tail. We show that the prescription works well in the parameter regime that has been currently explored in simulations. We then use Fisher matrix techniques to compare our prescription with an extrapolated fit to $N$-body simulations, which has recently been used to obtain constraints from data collected by the South Pole Telecope. We show that for the current data, both prescriptions would lead to statistically consistent constraints. As the data improve, however, there is a possibility of introducing a statistically significant bias in the constraints due to the choice of prescription, especially if non-Gaussianity is scale dependent and becomes relatively large on cluster scales. It would then be necessary to test the accuracy of the prescriptions in $N$-body simulations that can probe clusters with high masses and redshifts in the presence of large non-Gaussianity.
\end{abstract}

\section{INTRODUCTION}

One of the key aims in cosmology is to determine how much non-Gaussianity there was in the primordial curvature fluctuations. Canonical single field inflation with a smooth potential predicts that primordial non-Gaussianity (NG) should be extremely small [1, 2]. Detecting 1 an appreciable amount of primordial NG could therefore rule out these models. Measurements of the cosmic microwave background (CMB) [4] and the scale-dependent halo bias [5] provide good constraints on primordial NG on scales of about $k \sim 0.05 h^{-1} \mathrm{Mpc}$. Although the Planck experiment will improve the range of scales constrained [ $\left[\right.$, , 7], the primary CMB will alway be limited by foregrounds to $k \lesssim 0.2 h^{-1} \mathrm{Mpc}$. Since it is possible for primordial NG to be quite strongly scale dependent [8, 9], it is important to constrain it on as wide a range of scales as possible, with complementary probes when possible. Number counts of galaxy clusters provide such a probe on smaller scales [7] (see also Refs. 10-12]). There have been several recent attempts at using massive high-redshift clusters to constrain primordial NG [13 17]. Williamson et al. (W11) [17] analysed a subset of the clusters detected by the South Pole Telescope (SPT), using a likelihood analysis which carefully accounts for issues such as the survey selection function. They find a posterior probability distribution for the standard non-Gaussianity parameter $f_{\mathrm{NL}}$, and their quoted result is $f_{\mathrm{NL}}=20 \pm 450$ at $68 \%$ confidence.

A key ingredient in any such analysis is the chosen prescription for the non-Gaussian halo mass function. Given a mass function, one can construct a likelihood for the data by computing the expected number of clusters in a given mass and redshift range, as an integral of the mass function. The W11 analysis uses the prescription of Dalal et al. (D08) [18], which is essentially a fit to $N$-body simulations. As we will see however, the W11 likelihood function probes a region of parameter space (the extreme tail of the mass function) which has not been calibrated by D08 (nor indeed, by anyone else). Additionally, all the other currently available prescriptions [7, 19 22] formally break down in this extreme region.

\footnotetext{
*aparanja@ictp.it

$\dagger$ cxg@astro.ox.ac.uk

$\ddagger$ shaun.hotchkiss@helsinki.fi

1 See Ref. 3] for a review.
} 
It is therefore interesting to ask whether one can obtain any analytical understanding of the mass function in its extreme tail, and whether the D08 prescription might be introducing a bias in the analysis. The main result of this work is a new prescription for the non-Gaussian mass function, which involves a resummation of an infinite perturbative series. The resulting expression compares well with the results of $N$-body simulations. More importantly, it does not formally break down in the region of parameter space that needs to be probed by the W11 data analysis, and can therefore be used to compare against the D08 prescription. Using a Fisher analysis, we find that for the current quality of data the two prescriptions give statistically identical results. With better quality data however, we show that there can be a statistically significant bias between the two methods, especially if $f_{\mathrm{NL}}$ on small scales is large. It would then be necessary to accurately calibrate the tail of the non-Gaussian mass function in simulations.

The paper is organised as follows : Section [I] motivates and introduces our new "resummed" prescription for the mass function, comparing it with other prescriptions, both in regimes which have been tested by simulations and in the extreme tail which has not. In section [II] we use a Fisher analysis to compare the error bars on $f_{\mathrm{NL}}$ from the D08 and resummed prescriptions, and analyse the level of bias between the two methods. We end with a brief discussion in section IV] Technical details of calculations have been relegated to the Appendix. Unless otherwise specified, we assume a flat $\Lambda$ CDM cosmology with parameter values compatible with WMAP7 [4] : $h=0.703$ with $H_{0}=100 h \mathrm{kms}^{-1} \mathrm{Mpc}^{-1}$ the Hubble constant, total matter density $\Omega_{m} h^{2}=0.134$, baryonic matter density $\Omega_{b} h^{2}=0.0227$, scalar spectral index $n_{s}=0.966$, and $\sigma_{8}=0.809$. Throughout we use the transfer function of Bardeen et al. 223], with a baryonic correction as prescribed in Ref. [24].

\section{NUMBER COUNTS IN THE EXTREME TAIL}

Within the paradigm of hierarchical structure formation, any physically acceptable mass function ${ }^{2}$ must be compatible with the fact that massive objects tend to form late in the cosmic evolution. Mass functions derived assuming Gaussian initial conditions 25 27] easily fit the bill by virtue of being monotonically decreasing at large masses and redshifts. The situation with non-Gaussian initial conditions is not as clean however. There are several prescriptions in the literature for theoretically calculating the non-Gaussian mass function [7, 19 22]. All of these rely on a perturbative treatment of non-Gaussianity and assume a condition which is at least as strong as $|\epsilon \nu|<1$ (see D'Amico et al. (D11) [21] for a detailed comparison), where $\epsilon \sim f_{\mathrm{NL}} \sqrt{A}$ is a small parameter controlling the non-Gaussianity $\left(A \sim 10^{-9}\right.$ being the power spectrum normalisation) and

$$
\nu(M, z) \equiv \frac{\delta_{c}}{\sigma_{M}} \frac{D(0)}{D(z)},
$$

with the threshold $\delta_{c}=1.686$ (for spherical collapse, but see below), the linear growth rate $D(z)$, and the variance $\sigma_{M}^{2} \equiv\left\langle\hat{\delta}_{M}^{2}\right\rangle$ of the linearly extrapolated density field smoothed on scale $R=(3 M / 4 \pi \bar{\rho})^{1 / 3}$. While several of these mass functions have been tested in $N$-body simulations and do reasonably well in their regime of validity, when extrapolated to $|\epsilon \nu|>1$ they lead to unphysical results (e.g. the Matarrese et al. (MVJ) [19] result becomes imaginary for $f_{\mathrm{NL}}>0$ when $\epsilon \nu \sim 3$ ). This would be an academic issue, were it not for the fact that the current quality of data requires predictions of number counts at combinations of $\left(f_{\mathrm{NL}}, M, z\right)$ values which lie squarely in the $|\epsilon \nu|>1$ corner [16]. To see this, note that e.g. the W11 analysis proceeds by calculating the joint probability, for a given $f_{\mathrm{NL}}$, of observing the ensemble of clusters in their sample, and of observing nothing else in the parameter range they explore. The large errors on $f_{\mathrm{NL}}(\sim 450)$ indicate that their likelihood is non-negligible at say $f_{\mathrm{NL}} \lesssim 750$. Fig. 1 shows $\varepsilon_{1} \nu$ as a function of mass at redshift $z=1.5$ for some representative $f_{\mathrm{NL}}$ values, where

$$
\varepsilon_{1} \equiv\left\langle\hat{\delta}_{M}^{3}\right\rangle / \sigma_{M}^{3},
$$

with $\varepsilon_{1} \simeq 3 \times 10^{-4} f_{\mathrm{NL}}$ for a WMAP7 cosmology, and we identify $\varepsilon_{1}$ with the small parameter $\epsilon$ mentioned above. (Throughout this paper we will use the local model of NG to compute $\varepsilon_{1}$, see e.g. D11 for details.) We see that for $f_{\mathrm{NL}}=750$ at this redshift, $\epsilon \nu \sim 1$ at $M \sim 5 \times 10^{14} h^{-1} M_{\odot}$, which is well inside the region explored by W11.

In contrast with the theoretical approaches, the D08 prescription constructs a non-Gaussian mass function by convolving a Gaussian mass function with a Gaussian probability distribution whose mean and variance depend on $f_{\mathrm{NL}}$. As it turns out, this prescription results in the only viable mass function currently on the market which remains stable at large $\left(f_{\mathrm{NL}}, M, z\right)$. Since the W11 analysis uses this prescription 3 , it at least does not suffer from the

\footnotetext{
${ }^{2}$ By mass function we mean $f_{\text {sky }}(d V / d z)(d n / d M)$; where $d n$ is the comoving number density of halos with masses in $(M, M+d M), f_{\text {sky }}$ is the fraction of sky observed and $d V$ is the volume element with $d V / d z=4 \pi H(z)^{-1}\left[\int_{0}^{z} d z^{\prime} H\left(z^{\prime}\right)^{-1}\right]^{2}$.

${ }^{3}$ Presumably W11 used the exact numbers which D08 quote for the parameters of their fit; this detail is not mentioned in W11.
} 


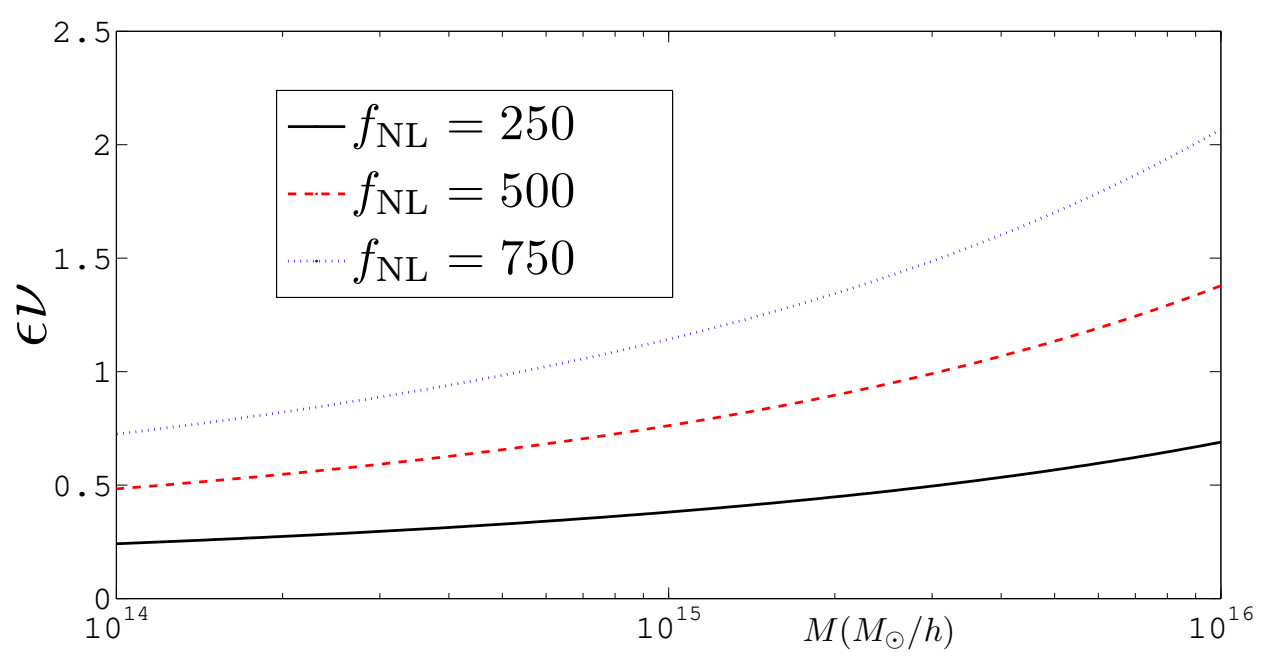

FIG. 1: The quantity $\varepsilon_{1} \nu$, where $\varepsilon_{1}$ and $\nu$ were defined in Eqns. (2) and (1) respectively. The curves are for redshift $z=1.5$ and three different values of $f_{\mathrm{NL}}$, all probed by the W11 likelihood analysis.

breakdown which the MVJ, LoVerde et al. (LMSV) 7] or LoVerde \& Smith (LS) [22] mass functions would encounter. There is however some cause for concern. Firstly, the actual parameter values D08 quote are a numerical fit to simulations which had considerably large Poisson errors and scatter at large $M$ and $z$ (see Fig.6 of D08). Subsequent simulations by Pillepich et al. (PPH) [28] indicate that this specific fit does not work very well at the low mass end either. The main problem however is that this fit applies in a mass and redshift regime where $|\epsilon \nu|$ is at most $\sim 0.5$, and there is no reason to expect it to work accurately when $\epsilon \nu>1$. One would therefore like to have some analytical understanding of this extreme tail of the mass function.

\section{A. A New Stable Non-Gaussian Mass Function}

In this work we present a new analytical non-Gaussian mass function which is stable in the $|\epsilon \nu|>1$ regime. As we show in the Appendix, under some technical assumptions regarding the form of primordial non-Gaussianity, which are inspired by a known perturbative hierarchy in the local model, the perturbative series appearing in the analysis can be resummed. The excursion set formalism then gives a mass function which is valid at arbitrarily large $\nu$ for a given positive $f_{\mathrm{NL}}$ (which can also be large, see below). The formal excursion set result needs to be modified in order to predict the correct Gaussian mass function when $f_{\mathrm{NL}} \rightarrow 0$. Assuming that the Gaussian mass function is well described by the Tinker et al. form [29], the "resummed" mass function we prescribe is

$$
\left.\frac{d n}{d M}\right|_{\text {Resum }}=\left.\frac{d n}{d M}\right|_{\text {Tinker }} \times \mathcal{R}_{\text {Resum }}
$$

where the ratio $\mathcal{R}_{\text {Resum }}$ is given by

$$
\mathcal{R}_{\text {Resum }}\left(M, z, f_{\mathrm{NL}}\right)=\left(1+\varepsilon_{1} \nu\right)^{-1 / 2} \exp \left[\frac{1}{2} \nu^{2}+\frac{1}{\varepsilon_{1}^{2}}\left(\varepsilon_{1} \nu-\left(1+\varepsilon_{1} \nu\right) \ln \left(1+\varepsilon_{1} \nu\right)\right)\right] .
$$

The expression is formally valid for $\left(1+\varepsilon_{1} \nu\right)>0$, and receives a relative correction of order $\mathcal{O}\left(\varepsilon_{1}^{2}\left(1+\varepsilon_{1} \nu\right)^{-1}\right)$ due to a saddle point approximation (see the Appendix for details). This shows that although $f_{\mathrm{NL}}$ cannot be made arbitrarily large for a given $\nu$, values of the order $f_{\mathrm{NL}} \lesssim 1000$ can be easily accomodated over the full $(M, z)$ range, and the saddle point approximation becomes increasingly accurate for large $(M, z)$. Finally, since the Tinker et al. mass function falls off like $\sim e^{-0.837 \delta_{c}^{2} / 2 \sigma^{2}}$, we need to redefine the $\nu$ that appears in $\mathcal{R}_{\text {Resum }}$ by replacing

$$
\delta_{c} \rightarrow \sqrt{q_{\text {Tinker }}} \delta_{c},
$$

in Eqn. (11) where $q_{\text {Tinker }}=0.837$. This ensures that the resulting mass function is still stable for arbitrarily large values of $\nu$. This correction is similar to the one first introduced by Grossi et al. [30] and can be motivated by appealing 

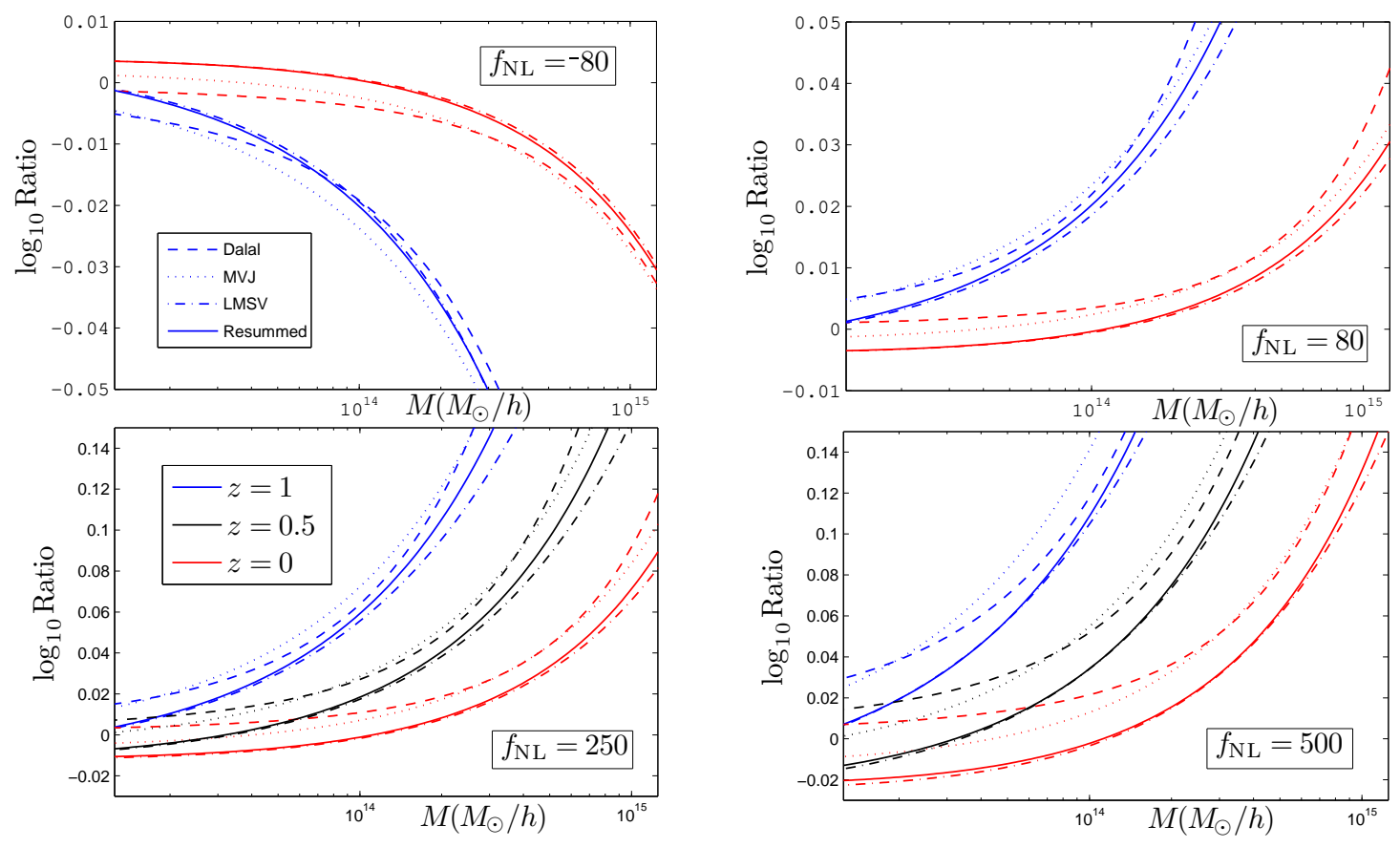

FIG. 2: Non-Gaussian ratios $\left.(d n / d M)\right|_{\mathrm{NG}} /\left.(d n / d M)\right|_{\text {Gauss }}$ as per different prescriptions, for values of $f_{\mathrm{NL}}$ tested in the PPH $N$-body simulations [28]. These plots use the same cosmology as used by PPH in their simulations (the WMAP5 values in Table 2 of PPH). The curves correspond to resummed with $q=q_{\text {Tinker }}=0.837$ (solid), LMSV 7$]$ with $q=0.79$ (dot-dashed), MVJ [19] with $q=0.79$ (dotted), and D08 [18] (dashed) calculated using the Tinker et al. [29] Gaussian mass function. The different sets of curves in each panel correspond to different redshifts. The lower panels show, from left to right, redshifts $z=1,0.5$ and 0 . The upper panels show $z=1$ and 0 , with $z=1$ corresponding to the lower set of curves in the left panel $\left(f_{\mathrm{NL}}=-80\right)$ and vice-versa in the right panel $\left(f_{\mathrm{NL}}=+80\right)$. We see that in this regime of parameter space, the resummed prescription (which has no free parameters once the Gaussian mass function is chosen) lies very close to LMSV with $q=0.79$. These plots can be directly compared with Fig. 5 of PPH, who showed that LMSV with $q=0.79$ fares very well compared to simulations. Consequently, so does the resummed prescription.

to a stochasticity in the spherical collapse barrier [31]. We emphasize that this modification, and the specific value $q_{\text {Tinker }}$, is forced on us by our choice of the Gaussian mass function - we have no free parameters to play with in our derivation.

Fig. 2 shows the non-Gaussian ratios according to various prescriptions, for values of $f_{\mathrm{NL}}$ tested in the PPH simulations. For better comparison with PPH, these plots assume the same cosmology that PPH used in their simulations (the WMAP5 values in their Table 2). We see that in the range plotted, the ratio $\mathcal{R}_{\text {Resum }}$ is very close to the LMSV ratio $\mathcal{R}_{\text {LMSV }}$ (which is plotted using a $\nu$ corrected with $q=0.79$ ). This can be understood analytically since in the regime $\epsilon \nu^{3}<1, \mathcal{R}_{\text {Resum }}$ and $\mathcal{R}_{\text {LMSV }}$ become formally identical when truncated at order $\mathcal{O}(\epsilon \nu)$. The residual difference is due partly to higher order terms and partly to the different values of the $q$-correction. Fig. 2 can be directly compared with Fig. 5 of $\mathrm{PPH}$, who showed that $\mathcal{R}_{\mathrm{LMSV}}$ with $q=0.79$ performs very well compared to simulations in this range. Consequently, so does our resummed ratio.

For large negative $f_{\mathrm{NL}}$ (or large $\nu$ ) such that $\epsilon \nu<-1$, our prescription breaks down. Nevertheless, there is a very simple way of extending the mass function to this regime. We notice that for $|\epsilon \nu| \ll 1$, it is approximately true that $\mathcal{R}_{\text {Resum }}\left(M, z,-f_{\mathrm{NL}}\right) \simeq \mathcal{R}_{\text {Resum }}\left(M, z, f_{\mathrm{NL}}\right)^{-1}$. We therefore define the resummed ratio for negative $f_{\mathrm{NL}}$ and arbitrary $\nu$ by

$$
\mathcal{R}_{\operatorname{Resum}}\left(M, z,-f_{\mathrm{NL}}\right) \equiv\left(\mathcal{R}_{\text {Resum }}\left(M, z, f_{\mathrm{NL}}\right)\right)^{-1} ; f_{\mathrm{NL}}>0 .
$$

Of course this is completely ad hoc, but we see from Fig. 2 that the prescription compares extremely well with $\mathcal{R}_{\text {LMSV }}$ at values of $f_{\mathrm{NL}}<0$ that were tested in the PPH simulations. It will be very interesting to see how the resummed ratio compares with simulations at high masses and redshifts, for both positive and negative $f_{\mathrm{NL}}$ values.

\footnotetext{
4 The MVJ and LMSV ratios in our language are respectively given by $\mathcal{R}_{\mathrm{MVJ}}=\left(1-\frac{1}{3} \varepsilon_{1} \nu\right)^{-1 / 2} e^{\varepsilon_{1} \nu^{3} / 6}\left(1-\frac{1}{2} \varepsilon_{1} \nu\left(1-\frac{2}{3} \dot{\varepsilon}_{1}\right)\right)$ and $\mathcal{R}_{\mathrm{LMSV}}=1+\frac{1}{6} \varepsilon_{1} \nu^{3}\left(1-\frac{1}{\nu^{2}}\left(3-2 \dot{\varepsilon}_{1}\right)-\frac{2}{\nu^{4}} \dot{\varepsilon}_{1}\right)$, where $\dot{\varepsilon}_{1} \equiv d \ln \varepsilon_{1} / d \ln \sigma^{2}$.
} 


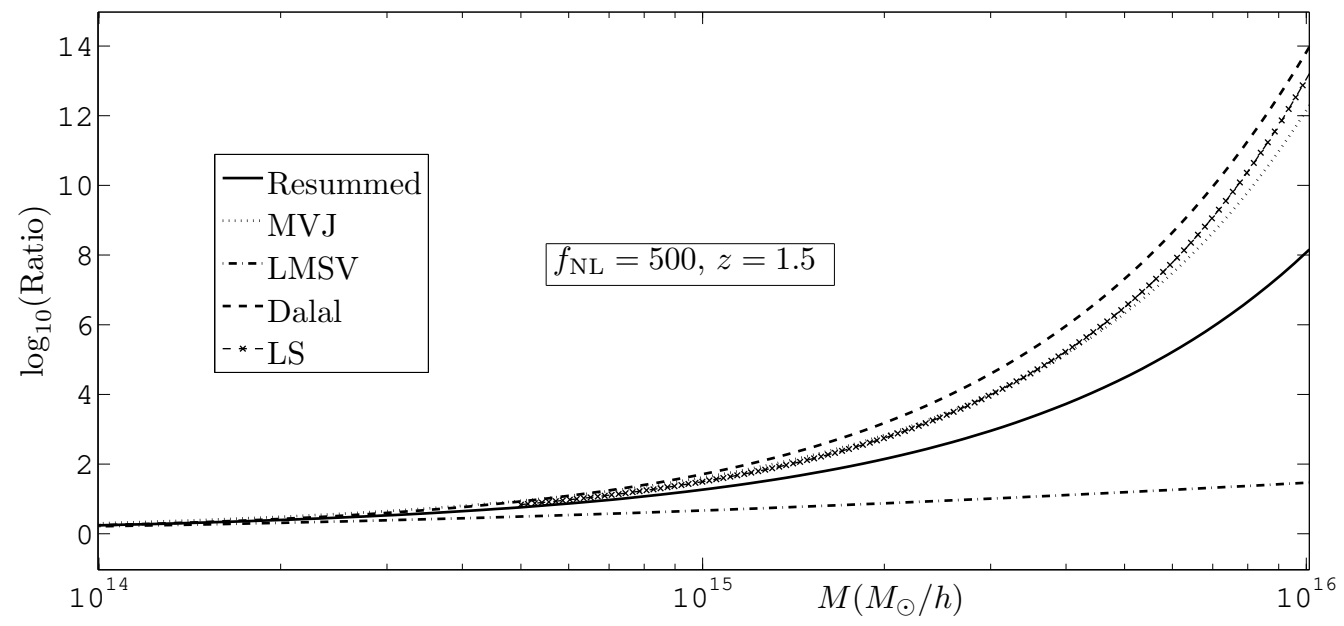

FIG. 3: Non-Gaussian ratios $\left.(d n / d M)\right|_{\mathrm{NG}} /\left.(d n / d M)\right|_{\text {Gauss }}$ as per different prescriptions, for $f_{\mathrm{NL}}=500$ and $z=1.5$. Comparing with Fig. 1 we see that the curves now enter the regime $\epsilon \nu>1$. The curves correspond to resummed with $q=q_{\text {Tinker }}=0.837$ (solid), LMSV (dot-dashed) and MVJ (dotted) with $q=0.79$, D08 (dashed) with the Tinker et al. Gaussian, and the "log-Edgeworth" ratio prescribed by LS [22] (cross-dashed). The D08 prescription continues to systematically predict a larger number of massive halos than the resummed.

\section{CONSTRAINTS ON $f_{\mathrm{NL}}$}

Fig. 2 also shows that for a given positive $f_{\mathrm{NL}}$, the D08 ratio $\mathcal{R}_{\text {Dalal }}$ is consistently higher than $\mathcal{R}_{\text {Resum }}$ and therefore predicts more high mass halos. This trend remains true even in the regime $\epsilon \nu>1$, as Fig. 3 shows. It is then worth asking how sensitive the analysis of clusters $a$ la W11 is to this difference 5 .

We use a Fisher analysis to make a "forecast" for the kind of data that SPT has already observed (specifically the subset used by W11). We set our fiducial cosmology to the WMAP7 values given earlier, with $f_{\mathrm{NL}}=0$. We marginalise over $\sigma_{8}$ with a WMAP7 prior, but assume perfect knowledge of all other cosmological parameters. This is not expected to significantly affect our conclusions. We bin in redshift between $0.3 \leq z \leq 2$, with a spacing $\Delta z=0.05$. The lower limit is the same used by W11, and we have checked that the analysis is insensitive to increasing the upper limit. We assume that the survey sees all clusters in any given redshift bin above a certain constant mass threshold $M_{\lim }$, which is approximately true for a Sunyaev-Zeldovich survey such as SPT [33]. We marginalise over $M_{\lim }$ with a lognormal prior of $30 \%$ in $M_{\text {lim }}$, in keeping with typical mass uncertainties quoted by W11. The fiducial value for $M_{\text {lim }}$ is chosen such that the total expected number of clusters with masses above $M_{\text {lim }}$ and at redshifts $z \geq 0.3$ in the fiducial cosmology, is roughly the same as in the W11 analysis, which is 26 . For $f_{\mathrm{NL}}=0$, (i.e. - using the Tinker et al. mass function) this gives us a fiducial value $M_{\text {lim }}=8.5 \times 10^{14} h^{-1} M_{\odot}$ for $f_{\text {sky }}=0.06$.

We then assume a joint likelihood given by a product of independent Poisson probabilities for each redshift bin 6 , and construct the Fisher matrix for parameters $\theta_{a}=\left(M_{\mathrm{lim}}, \sigma_{8}, f_{\mathrm{NL}}\right)[35]$

$$
F_{a b}=\sum_{i=1}^{n_{\text {bins }}} \frac{1}{\mu_{i}} \frac{\partial \mu_{i}}{\partial \theta_{a}} \frac{\partial \mu_{i}}{\partial \theta_{b}}
$$

where $\mu_{i}$ is the expected number of clusters in the $i^{\text {th }}$ redshift bin, evaluated as the integral of $(d n / d M)(d V / d z)$ over this bin, for masses above $M_{\mathrm{lim}}$. The results are in Table I, which shows the marginalised errors on $f_{\mathrm{NL}}$. We see that the D08 mass function results in a marginal error $\sigma_{f_{\mathrm{NL}}} \simeq 300$, which can be compared with the error quoted by W11 which is 450 . This shows that our analysis works; the fact that our marginal error for D08 is smaller, is

${ }^{5}$ We note that in contrast to W11, earlier analyses [15, 16] showed a significant tension with standard $\Lambda$ CDM. It would then seem to be more interesting to perform our analysis on the set of clusters used in these analyses rather than W11. In fact, these analyses can be shown to have used a biased statistic, and removing this bias makes them consistent with the analysis of W11 [32]. We ultimately choose to study W11 because we find it easier to approximate the selection function for the Sunyaev-Zeldovich clusters measured by SPT.

${ }^{6}$ This is similar to what is done by W11, who use a product of independent Poisson probabilities over a number of bins in the space of redshift and detection significance which is roughly their mass proxy. As can be seen from the methods of Ref. 34], sample covariance should be negligible for this survey. 


\begin{tabular}{|c|c|c|}
\hline \multicolumn{3}{|c|}{ Marginal $\sigma_{f_{\mathrm{NL}}}$, W11 equivalent } \\
\hline Prescription & $f_{\mathrm{NL}}=0$ & $f_{\mathrm{NL}}=500$ \\
\hline \hline D08 & 298 & 463 \\
\hline Resummed & 457 & 599 \\
\hline MVJ & 487 & 288 \\
\hline LMSV & 498 & 1366 \\
\hline
\end{tabular}

TABLE I: The marginal Fisher error $\sigma_{f_{\mathrm{NL}}}$ as per different prescriptions, for two different choices of fiducial $f_{\mathrm{NL}}$, for a survey that is approximately equivalent to the subset of the SPT clusters analysed in W11 (see text for details). The marginalisation is over $\sigma_{8}$ with a WMAP7 prior and the threshold mass $M_{\text {lim }}$ with a $30 \%$ lognormal prior (see text). We see that the resummed and D08 prescriptions predict comparable errors in all cases, while the MVJ and LMSV predict significantly different values. See text for a discussion.

\begin{tabular}{|c|c|c|c|c|}
\hline \multicolumn{5}{|c|}{$\sigma_{f_{\mathrm{NL}}}$, Planck-like } \\
\hline & \multicolumn{2}{|c|}{ Conditional } & \multicolumn{2}{c|}{ Marginal } \\
\hline Prescription & $f_{\mathrm{NL}}=0$ & $f_{\mathrm{NL}}=500$ & $f_{\mathrm{NL}}=0$ & $f_{\mathrm{NL}}=500$ \\
\hline \hline D08 & 9.1 & 9.2 & 45.7 & 77.9 \\
\hline Resummed & 11.0 & 11.0 & 64.0 & 80.5 \\
\hline MVJ & 10.8 & 6.6 & 72.4 & 41.7 \\
\hline LMSV & 12.0 & 16.1 & 69.6 & 310 \\
\hline
\end{tabular}

TABLE II: Fisher errors on $f_{\mathrm{NL}}$ for a final Planck-like survey, with a $2 \%$ prior on $\sigma_{8}$ and a fiducial threshold $M_{\text {lim }}=5 \times 10^{14} h^{-1} M_{\odot}$ with a $10 \%$ lognormal prior and $f_{\text {sky }}=0.8$. The values under "conditional" assume perfect knowledge of all parameters except $f_{\mathrm{NL}}$, and represent what is possible if parameter degeneracies are broken using measurements of say, the clustering of clusters 10 12].

consistent with our simplifying assumptions regarding the cosmological parameters and selection function. The errors predicted by the resummed mass function are comparable to that predicted by the D08 prescription, indicating that using either prescription would lead to similar results. Since the errors are large however, one might wonder if the

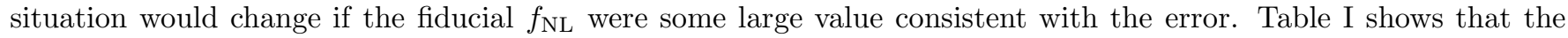
predicted errors from the D08 and resummed mass functions are still comparable at a fiducial $f_{\mathrm{NL}}=500$ (in this case we used a fiducial $M_{\lim }=1.05 \times 10^{15} h^{-1} M_{\odot}$ to get a total expected number close to 26 ).

Table \] shows the results of a Fisher forecast for a final Planck-like SZ survey [36], assuming a $2 \%$ prior on $\sigma_{8}$, a threshold $M_{\text {lim }}=5 \times 10^{14} h^{-1} M_{\odot}$ with a $10 \%$ prior, and binning in redshift between $0.1 \leq z \leq 2$ with a spacing $\Delta z=0.05$ and $f_{\text {sky }}=0.8$. In this case we also display the conditional error (i.e. - assuming perfect knowledge of other parameters), as being representative of what can be achieved when parameter degeneracies are broken using measurements of say, the clustering of clusters $10-12$. We see that the predicted conditional and marginal errors for $f_{\mathrm{NL}}$ are again comparable for the two mass function: 7 .

It appears therefore, that using the D08 fit at $\epsilon \nu>1$, as opposed to a theoretically motivated mass function such as the resummed, does not introduce a significant effect in the error predicted for $f_{\mathrm{NL}}$ from number counts of clusters. However, since the actual number of halos predicted at any given $f_{\mathrm{NL}}$ is different for these two mass functions, we must also worry about a possible bias in the central value of $f_{\mathrm{NL}}$. Estimating this properly would require a full-fledged Monte Carlo analysis that accurately accounts for survey selection functions, which is work in progress. For now, we perform a cruder analysis. For parameters corresponding to the final Planck-like survey mentioned above, we assume a fiducial cosmology of the WMAP7 $\Lambda$ CDM, but this time with a non-zero value of $f_{\mathrm{NL}}=f_{\mathrm{NL} *}$ using the D08 prescription. We then "analyse data" using the resummed prescription, by constructing an approximate likelihood

\footnotetext{
7 This might seem surprising given the difference between the magnitudes of these mass functions. In fact, it might appear from Fig. 3 that the MVJ prescription should be closer to D08. Note however, that the Fisher matrix (7) depends not only on the integrated mass function, but also on its derivative with respect to $f_{\mathrm{NL}}$, leading to a complicated interplay which is difficult to predict simply by examining plots of the mass function or non-Gaussian ratio.
} 


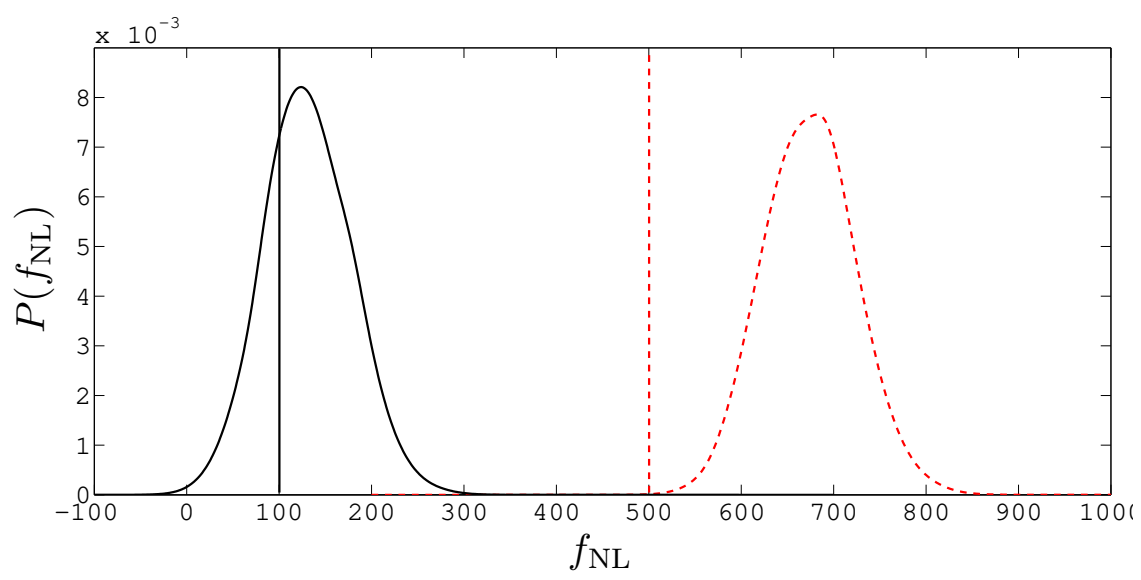

FIG. 4: Testing for bias between D08 and resummed for a final Planck like survey : Posterior probability distribution $p\left(f_{\mathrm{NL}} \mid f_{\mathrm{NL}}\right)$ computed by marginalising the approximate likelihood (8) for the D08 "data" given the resummed "model", over $M_{\text {lim }}$ with a $10 \%$ lognormal prior. The curves are for $f_{\mathrm{NL}_{*}}=100$ (solid,black) and $f_{\mathrm{NL}_{*}}=500$ (dashed,red). The corresponding vertical lines mark the value of the respective $f_{\mathrm{NL}_{*}}$. We see a statistically significant relative bias between the resummed and D08 prescriptions for large $f_{\mathrm{NL}_{*}}$, while at smaller $f_{\mathrm{NL}_{*}}$ the prescriptions are statistically consistent with each other.

given by

$$
\mathcal{L}\left(f_{\mathrm{NL}}, M_{\mathrm{lim}}\right) \propto \exp \left[-\frac{1}{2} \sum_{i=1}^{n_{\text {bins }}}\left(\frac{\left(\mu_{i}^{\text {Resum }}-\mu_{i *}^{\text {Dalal }}\right)^{2}}{\mu_{i}^{\text {Resum }}}+\ln \mu_{i}^{\text {Resum }}\right)\right],
$$

where $\mu_{i *}^{\text {Dalal }}=\mu_{i}^{\text {Dalal }}\left(f_{\mathrm{NL} *}, M_{\text {lim* }}\right)$ is the fiducial expectation value in the $i^{\text {th }}$ bin, which we treat as "data", and $\mu_{i}^{\text {Resum }} \stackrel{{ }^{*}}{=} \mu_{i}^{\text {Resum }}\left(f_{\mathrm{NL}}, M_{\mathrm{lim}}\right)$ is the model expectation value computed using the resummed prescription at the $\left(f_{\mathrm{NL}}, M_{\mathrm{lim}}\right)$ values being analysed. (We keep $\sigma_{8}$ fixed in this exercise.) We marginalise this likelihood over $M_{\text {lim }}$ with a $10 \%$ lognormal prior (assuming a flat prior on $f_{\mathrm{NL}}$ ), to obtain a posterior probability distribution for $f_{\mathrm{NL}}$. Fig. 4 shows the results for $f_{\mathrm{NL} *}=100$ and 500. We see that for $f_{\mathrm{NL} *}=100$, the resummed posterior $p\left(f_{\mathrm{NL}} \mid f_{\mathrm{NL} *}=100\right)$ peaks close to $f_{\mathrm{NL} *}$ and there is no statistically significant bias; $f_{\mathrm{NL} *}$ lies less than one standard deviation away from the peak value. For $f_{\mathrm{NL}_{*}}=500$ on the other hand, the value $f_{\mathrm{NL}_{*}}$ lies far in the tail (more than 4 standard deviations away from the peak) of the distribution $p\left(f_{\mathrm{NL}_{\mathrm{L}}} \mid f_{\mathrm{NL}_{*}}=500\right)$, indicating a statistically significant bias. A similar calculation for the SPT equivalent parameter values used in Table $\amalg$ shows that with the current data there would be no detectable difference between analyses based on the resummed or D08 prescriptions. We can conclude that systematic effects from the choice of non-Gaussian mass function could become important as the data improves, if non-Gaussianity is scale dependent and results in a large $f_{\mathrm{NL}}$ on cluster scales. In this case it would be necessary to properly calibrate the non-Gaussian mass function in its extreme tail.

\section{SUMMARY \& CONCLUSIONS}

Number counts of clusters offer a very interesting probe of the extreme tail of the mass function of collapsed objects, and thereby the statistics of the primordial curvature fluctuations. In this work we developed a new prescription for calculating the modification of the mass function in the presence of primordial non-Gaussianity (Eqns. (3) and (44)). The key features of this "resummed" prescription are that it is theoretically well motivated (as opposed to a fit to simulations like D08 [18]) as well as stable in the extreme region $|\epsilon \nu|>1$ (unlike all the other currently available theoretical prescriptions [7, 19 22]). We also showed that this resummed prescription compares very well against the results of $N$-body simulations in the literature (see Fig. 2), despite having no free parameters left once its Gaussian limit is fixed as the Tinker et al. [29] mass function.

While this might appear to be an academic issue, we showed that the current quality of data forces any likelihood analysis to enter the regime $|\epsilon \nu|>1$. It is then important to analyse how far one can trust the chosen prescription for the non-Gaussian mass function. The specific example we dealt with was the W11 [17] analysis of a subset of the current SPT clusters, which used the D08 prescription to compute the likelihood. We used Fisher matrix techniques to analyse the consequences of changing the prescription from D08 to resummed, and found that for the current 
data, both prescriptions are expected to give approximately identical results. As the data improve however, there is a possibility of introducing a statistically significant bias in the analysis, due to the choice of prescription. It will then become important to evaluate which is the better method, which will require improved $N$-body simulations. This would be especially true if non-Gaussianity is scale dependent and results in a large value of $f_{\mathrm{NL}}$ on cluster scales [8, [9]. One would then need to calibrate the mass function using $N$-body simulations which may have to be specially tailored (with larger particle size, say) to probe clusters with high masses, at large redshifts and in the presence of large $f_{\mathrm{NL}}$.

\section{ACKNOWLEDGMENTS}

We thank L. Sriramkumar and other organisers of the Primordial Features and Non-Gaussianities (PFNG) meeting at the Harish-Chandra Research Institute in December 2010 where this work was initiated. We also thank Ravi Sheth for helpful discussions. CG is funded by the Beecroft Institute for Particle Astrophysics and Cosmology. SH is supported by the Academy of Finland grant 131454 .

\section{Appendix A: The Resummed Mass Function}

In this Appendix we sketch the derivation of the resummed mass function presented in the text, describing all the approximations that enter. The non-Gaussian halo mass function derived from the excursion set formalism for spherical collapse is

$$
\left.\frac{d n}{d M}\right|_{\mathrm{NG}, \mathrm{exc}}=\frac{\bar{\rho}}{M^{2}} f_{\mathrm{NG}, \operatorname{exc}}\left|\frac{d \ln \sigma}{d \ln M}\right|
$$

where the multiplicity $f_{\mathrm{NG}, \text { exc }}$ for large masses is given by (see D11 [21])

$$
f_{\mathrm{NG}, \operatorname{exc}}\left(\nu, \varepsilon_{1}, \varepsilon_{2}, \ldots\right)=\sqrt{\frac{2}{\pi}} \nu \exp \left[\sum_{n=3}^{\infty} \frac{(-1)^{n}}{n !} \varepsilon_{n-2} \partial_{\nu}^{n}\right]\left\{e^{-\frac{1}{2} \nu^{2}}+\ldots\right\},
$$

where $\nu(M, z)$ was defined in Eqn. (11) and $\varepsilon_{n-2} \equiv\left\langle\hat{\delta}_{M}^{n}\right\rangle / \sigma_{M}^{n}$ are the normalised connected moments of the linearly extrapolated, smoothed density field. This expression assumes that the functions $\varepsilon_{j}$ are all constant with scale in the regime of interest, which is reasonable at least in the local model (see e.g. Fig.1 of D11). If $f_{\mathrm{NL}}$ is the only NG parameter allowed, then these are perturbatively ordered : $\varepsilon_{j} \sim\left(f_{\mathrm{NL}} \sqrt{A}\right)^{j}$, with $A$ the normalisation of the power spectrum. The ellipsis in Eqn. (A2) indicates terms arising from "unequal-time" correlations as discussed in Refs. [20, 21]. In the large mass (or large $\nu$ ) limit, these will be of the form $\sim e^{-\frac{1}{2} \nu^{2}} \mathcal{O}(\epsilon \nu)$. Experience with the calculation of D11 indicates that the action of the exponential derivative on all these terms will result in a single common exponential prefactor multiplying a series expansion in $\epsilon \nu$. When $|\epsilon \nu| \sim 1$, the polynomial-type terms should resum, but it is hard (if not impossible) to predict the resummed form. We will therefore ignore these terms and concentrate on the exponential, with the post hoc justification that the approximations appear to work very well at intermediate masses and redshifts where non-Gaussian $N$-body simulations have been performed.

We are then after the quantity

$$
\begin{aligned}
f_{\mathrm{NG}, \operatorname{exc}}\left(\nu, \varepsilon_{1}, \varepsilon_{2}, \ldots\right) & \approx \sqrt{\frac{2}{\pi}} \nu \exp \left[\sum_{n=3}^{\infty} \frac{(-1)^{n}}{n !} \varepsilon_{n-2} \partial_{\nu}^{n}\right] e^{-\frac{1}{2} \nu^{2}} \\
& =\sqrt{\frac{2}{\pi}} \nu \int_{-\infty}^{\infty} \frac{d \lambda}{\sqrt{2 \pi}} e^{\phi(\lambda)}
\end{aligned}
$$

where we defined

$$
\phi(\lambda) \equiv i \lambda \nu+\sum_{n=2}^{\infty} \frac{(-i \lambda)^{n}}{n !} \varepsilon_{n-2} \quad ; \quad \varepsilon_{0} \equiv 1
$$

The integral in Eqn. (A3) can in principle be done using a saddle point approximation with calculable corrections, e.g. along the lines presented in D11. Unfortunately, as D11 showed, a perturbative treatment will necessarily require 
the condition $|\epsilon \nu|<1$, which is not sufficient for our purpose. Going beyond this technical barrier requires knowledge of an infinite number of connected moments of the density field. A key simplification occurs if we assume (inspired by the heirarchy $\left.\varepsilon_{j} \sim\left(f_{\mathrm{NL}} \sqrt{A}\right)^{j}\right)$ the exact relations

$$
\varepsilon_{j}=\epsilon^{j} ; \epsilon \equiv \varepsilon_{1} .
$$

One could treat this as defining a specific model of non-Gaussianity. Throughout, we will numerically compute $\varepsilon_{1}$ as in the local model of NG. The series in $\phi(\lambda)$ can then be re-arranged to bring the function into closed form,

$$
\phi(\lambda)=i \lambda \nu+\frac{1}{\epsilon^{2}}\left(e^{-i \lambda \epsilon}+i \lambda \epsilon-1\right),
$$

with derivatives

$$
\phi^{\prime}(\lambda)=i\left(\nu+\frac{1}{\epsilon}\left(1-e^{-i \lambda \epsilon}\right)\right) \quad ; \quad \phi^{\prime \prime}(\lambda)=-e^{-i \lambda \epsilon} .
$$

A saddle point now exists at $\lambda=\lambda_{*}$ where $\phi^{\prime}\left(\lambda_{*}\right)=0$, i.e.

$$
e^{-i \lambda_{*} \epsilon}=1+\epsilon \nu,
$$

provided we have $\phi^{\prime \prime}\left(\lambda_{*}\right)=-(1+\epsilon \nu)<0$, i.e. if $\epsilon \nu>-1$. The leading saddle point result for the multiplicity follows from setting $\int d \lambda / \sqrt{2 \pi} e^{\phi(\lambda)}=e^{\phi\left(\lambda_{*}\right)}\left(\left|\phi^{\prime \prime}\left(\lambda_{*}\right)\right|\right)^{-1 / 2}$, which gives

$$
\begin{aligned}
f_{\mathrm{NG}, \operatorname{exc}}(\nu, \epsilon) & \approx \sqrt{\frac{2}{\pi}} \nu(1+\epsilon \nu)^{-1 / 2} \exp \left[\frac{1}{\epsilon^{2}}(\epsilon \nu-(1+\epsilon \nu) \ln (1+\epsilon \nu))\right] \\
& \equiv f_{\mathrm{NG}, \mathrm{app}}(\nu, \epsilon),
\end{aligned}
$$

the subscript "app" reminding us of the approximations involved in the derivation.

One can also estimate the error involved in the saddle point approximation. The calculation proceeds along the lines discussed in Appendix D of D11, by first using the series representation of $\phi(\lambda)$ in the integral of $e^{\phi(\lambda)}$, and then Taylor expanding to get (after a change of variables) the exact result

$$
\int_{-\infty}^{\infty} \frac{d \lambda}{\sqrt{2 \pi}} e^{\phi(\lambda)}=e^{\phi\left(\lambda_{*}\right)}(1+\epsilon \nu)^{-1 / 2} \int_{-\infty}^{\infty} \frac{d y}{\sqrt{2 \pi}} e^{-\frac{1}{2} y^{2}}\left[1+\frac{(-i y)^{3}}{3 !} \tau^{1 / 2}+\frac{(-i y)^{4}}{4 !} \tau+\frac{1}{2 !}\left(\frac{(-i y)^{3}}{3 !}\right)^{2} \tau+\ldots\right],
$$

where $\tau \equiv \epsilon^{2} /(1+\epsilon \nu)>0$. Clearly, terms involving half-integer powers of $\tau$ will not contribute, and hence the relative correction to Eqn. (A9) is of order $\mathcal{O}(\tau)$ and is calculable in principle to arbitrary order in $\tau$. Since $\epsilon \sim 3 \times 10^{-4} f_{\mathrm{NL}}$, the approximation remains valid even for considerably large positive values of $f_{\mathrm{NL}}$, and becomes increasingly better for large $\nu$. Negative $f_{\mathrm{NL}}$ values do not fare as well though, due to the restriction $\epsilon \nu>-1$. See the main text however for a simple way of extending the mass function to $f_{\mathrm{NL}}<0$, which appears to work well in the regime where $N$-body simulations have been performed.

It is straightforward to check that for fixed $f_{\mathrm{NL}}$, the multiplicity $f_{\mathrm{NG} \text {,app }}(\nu, \epsilon)$ is monotonically decreasing with $\nu$ at large $\nu$, and the mass function is therefore stable in its extreme tail, as needed. One can also easily check (using $\left.\ln (1+x)=-\sum_{n=1}^{\infty}(-x)^{n} / n\right)$ that the limit $\epsilon \rightarrow 0$ recovers the Gaussian Press-Schechter result,

$$
\lim _{\epsilon \rightarrow 0} f_{\mathrm{NG}, \mathrm{app}}(\nu, \epsilon)=\sqrt{\frac{2}{\pi}} \nu e^{-\frac{1}{2} \nu^{2}} \equiv f_{\mathrm{PS}}(\nu) .
$$

In fact, retaining the leading terms in $\epsilon$ recovers the MVJ exponential prefactor

$$
f_{\mathrm{NG}, \text { app }}(\nu, \epsilon) \rightarrow \sqrt{\frac{2}{\pi}} \nu e^{-\frac{1}{2} \nu^{2}\left(1-\frac{1}{3} \epsilon \nu+\ldots\right)}(1+\mathcal{O}(\epsilon \nu)) .
$$

Since the Press-Schechter result is known to perform badly when compared with $N$-body simulations, we will follow the usual practice in the literature and prescribe a ratio of non-Gaussian and Gaussian mass functions. Assuming that the Gaussian mass function is well described by the Tinker et al. form [29], the final mass function to use is given by Eqn. (3), with a modified definition of $\nu$ as discussed in the main text.

[1] J. M. Maldacena, JHEP 0305, 013 (2003) arXiv:astro-ph/0210603. 
[2] V. Acquaviva, N. Bartolo, S. Matarrese and A. Riotto, Nucl. Phys. B 667, 119 (2003) arXiv:astro-ph/0209156.

[3] V. Desjacques and U. Seljak, Class. Quant. Grav. 27, 124011 (2010) arXiv:1003.5020 [astro-ph.CO]|.

[4] E. Komatsu et al. [ WMAP Collaboration ], Astrophys. J. Suppl. 192, 18 (2011). arXiv:1001.4538 [astro-ph.CO]].

[5] A. Slosar, et al., JCAP 0808 (2008) 031 arXiv:0805.3580 [astro-ph]].

[6] E. Sefusatti, et al., JCAP 0912, 022 (2009). arXiv:0906.0232 [astro-ph.CO]].

[7] M. LoVerde, A. Miller, S. Shandera and L. Verde, JCAP 0804 (2008) 014 [arXiv:0711.4126 [astro-ph]].

[8] A. Riotto, M. S. Sloth, Phys. Rev. D83, 041301 (2011). arXiv:1009.3020 [astro-ph.CO]].

[9] C. T. Byrnes, S. Nurmi, G. Tasinato, D. Wands, JCAP 1002, 034 (2010). arXiv:0911.2780 [astro-ph.CO]].

[10] C. Fedeli, C. Carbone, L. Moscardini and A. Cimatti, arXiv:1012.2305 [astro-ph.CO].

[11] C. Cunha, D. Huterer and O. Dore, Phys. Rev. D 82 (2010) 023004 arXiv:1003.2416 [astro-ph.CO]].

[12] B. Sartoris, et al., Mon. Not. Roy. Astron. Soc. 407, 2339 (2010), arXiv:1003.0841 [astro-ph.CO].

[13] R. Jimenez, L. Verde, Phys. Rev. D80, 127302 (2009). arXiv:0909.0403 [astro-ph.CO]].

[14] L. Cayón, C. Gordon, J. Silk, arXiv:1006.1950 [astro-ph.CO]], MNRAS in press.

[15] B. Hoyle, R. Jimenez, L. Verde, arXiv:1009.3884 [astro-ph.CO]].

[16] K. Enqvist, S. Hotchkiss, O. Taanila, arXiv:1012.2732 [astro-ph.CO]], JCAP in press.

[17] R. Williamson, et al., arXiv:1101.1290 [astro-ph.CO]].

[18] N. Dalal, O. Dore, D. Huterer and A. Shirokov, Phys. Rev. D 77 (2008) 123514 arXiv:0710.4560 [astro-ph]].

[19] S. Matarrese, L. Verde and R. Jimenez, Astrophys. J. 541 (2000) 10 arXiv:astro-ph/0001366].

[20] M. Maggiore, A. Riotto, Astrophys. J. 717, 526-541 (2010). arXiv:0903.1251 [astro-ph.CO]].

[21] G. D'Amico, M. Musso, J. Noreña, A. Paranjape, JCAP 1102, 001 (2011). arXiv:1005.1203 [astro-ph.CO]].

[22] M. LoVerde, K. M. Smith, arXiv:1102.1439 [astro-ph.CO]].

[23] J. M. Bardeen, J. R. Bond, N. Kaiser and A. S. Szalay, Astrophys. J. 304, 15 (1986).

[24] N. Sugiyama, Astrophys. J. Suppl. 100, 281 (1995) arXiv:astro-ph/9412025].

[25] W. H. Press andP. Schechter, Astrophys. J. 187, 425 (1974).

[26] J. R. Bond, S. Cole, G. Efstathiou and N. Kaiser, Astrophys. J. 379, 440 (1991).

[27] R. K. Sheth, G. Tormen, Mon. Not. Roy. Astron. Soc. 308, 119 (1999). astro-ph/9901122.

[28] A. Pillepich, C. Porciani, O. Hahn, Mon. Not. Royal Astron. Soc., 402, 191-206 (2010). arXiv:0811.4176 [astro-ph]].

[29] J. L. Tinker, et al., Astrophys. J. 688, 709-728 (2008). arXiv:0803.2706 [astro-ph]].

[30] M. Grossi, et al., Mon. Not. Roy. Astron. Soc. 382, 1261 (2007). arXiv:0707.2516] [astro-ph]].

[31] M. Maggiore, A. Riotto, Astrophys. J. 717, 515-525 (2010). arXiv:0903.1250 [astro-ph.CO]].

[32] S. Hotchkiss, arXiv:1105.3630 [astro-ph.CO]], JCAP in press.

[33] K. Vanderlinde, et al., Astrophys. J. 722, 1180-1196 (2010). arXiv:1003.0003 [astro-ph.CO]].

[34] W. Hu, A. V. Kravtsov, Astrophys. J. 584, 702-715 (2003). astro-ph/0203169.

[35] G. Holder, Z. Haiman, J. Mohr, Astrophys. J. 560 (2001) L111-L114. astro-ph/0105396].

[36] J. Geisbuesch, M. Hobson, Mon. Not. Roy. Astron. Soc. 382, 158 (2007), astro-ph/0611567. 